Revisión de tema

Vol. 19(2):142-150, agosto - noviembre 2016

\title{
Avances en el conocimiento de la prehabilitación y presentación del proyecto promoción de la ambulación precoz en pacientes de cirugía no cardíaca (PAMP)
}

Improvements in the knowledge of prehabilitation and presentation of the project: promotion of early ambulation in noncardiac surgery patients (PAMP)

\section{Avanços no Conhecimento da Pré-reabilitação e Apresentação do Projeto Promoção da deambulação precoce em pacientes com cirurgia não cardíaca (PAMP)}

\author{
Olga Lucía Cortés, Enf., MSc., PhD.* \\ Claudia Patricia Becerra-Cristancho, ** \\ Adriana Lisette Hine-Sanabria, Enf.***
}

\author{
Skarlet Vásquez-Hernández, Enf.**** \\ Maribel Esparza-Bohórquez, Enf., MSc.***** \\ Ximena Acuña-Torres, Enf ******
}

\section{Resumen}

Introducción: La prehabilitación es un programa multidisciplinario que combina un conjunto de intervenciones (caminata, ejercicios aeróbicos, entrenamiento de los músculos inspiratorios, educación sobre nutrición y manejo psicológico) orientadas hacia el mejoramiento de la capacidad funcional de los pacientes antes de asumir cualquier tipo de cirugía. Su propósito es el de preparar físicamente a los pacientes que van a cirugía (especialmente población adulta y adulta mayor) y favorecer una rehabilitación rápida, la reducción de las complicaciones en el post operatorio, y una preparación adecuada para el egreso hospitalario. A pesar del conocimiento de la importancia de esta intervención poco se sabe sobre su beneficio en los resultados post operatorios de pacientes de cirugía no cardíaca. Objetivo: Describir la prehabilitación, los alcances de la evidencia científica en este tópico y presentar el proyecto promoción de la ambulación precoz en pacientes de cirugía no cardíaca (PAMP) como un modelo de investigación en cirugía no cardíaca y prehabilitacion. Metodología: Se realizó una revisión no sistemática de la literatura de los fundamentos de la prehabilitación, sus orígenes, tipos de intervenciones, importancia y su eficacia en la reducción de eventos en el post operatorio de pacientes de cirugía no cardíaca. Adicionalmente se llevó a cabo una revisión de la literatura que incluyó revisiones sistemáticas y metaanálisis de ensayos clínicos sobre intervenciones evaluadas sobre prehabilitación de cirugía no cardíaca. Finalmente, se describió el alcance del proyecto PAMP en todas sus fases y su progreso hacia la implementación de un ensayo clínico de prehabilitación en Colombia mediante el estudio PAMP fase

\footnotetext{
* Doctora en Enfermería. Investigador Asociado Departamento de Investigaciones. Fundación Cardioinfantil-Instituto de Cardiología. Bogotá, Colombia.

** Estudiante de Enfermería Práctica Electiva de Profundización. Fundación Cardioinfantil-Instituto de Cardiología. Bogotá, Colombia

*** Enfermera. Coordinadora de Estudio PAMP Fase II. Fundación Cardioinfantil Instituto de Cardiología. Bogotá, Colombia.

**** Enfermera. Investigadora Asociada Grupo Cardiología Preventiva Universidad Autónoma de Bucaramanga, Colombia.

***** Enfermera. Directora Departamento de Enfermería. Fundación Carlos Ardila Lülle. Foscal, Bucaramanga, Colombia.

****** Enfermera. Coordinadora de Estudio PAMP Fase II. Fundación Carlos Ardila Lülle. Foscal, Bucaramanga, Colombia.
}

Autor de Correspondencia: Olga Lucía Cortés. Enf, MSc, PhD. Cl. 163a \#13B-60, Fundación Cardioinfantil-Instituto de Cardiología. Bogotá, Colombia. Correo electrónico: olgacortesf@gmail.com 
II. Conclusiones: Se requiere implementar estrategias multidisciplinarias innovadoras que promuevan mejores resultados en el post operatorio de pacientes sometidos a cirugía no cardíaca. [Cortés OL, Becerra-Cristancho CP, Hine-Sanabria AL, Vásquez-Hernández SM. Avances en el conocimiento de la prehabilitación y presentación del proyecto promoción de la ambulación precoz en pacientes de cirugía no cardíaca (PAMP). MedUNAB 2016; 19(2): 142-150].

Palabras clave: Cirugía General; Período preoperatorio; Periodo posoperatorio; Anciano; Ejercicio.

\section{Abstract}

Introduction: Prehabilitation is a multidisciplinary program that combines a set of interventions (walking, aerobic exercises, inspiratory muscle training, nutrition education and psychological management) aimed at improving the functional capacity of patients before assuming any type of surgery. Its purpose is to physically prepare patients before going to surgery (especially the adult and older adult population) and favor rapid rehabilitation, reduction of postoperative complications, and adequate preparation for hospital discharge. Despite the knowledge of the importance of this intervention it is not a practice implemented and little is known about its benefit in the postoperative results of noncardiac surgery patients. Objective: To describe the prehabilitation and the scope of the scientific evidence in this topic. To present the PAMP project as a research model of prehabilitation in non-cardiac surgery. Methodology: We performed a non - systematic review of the literature on the fundamentals of prehabilitation, its origins, types of interventions, importance and its efficacy in the reduction of postoperative events in noncardiac surgery patients. Furthermore, we performed a literature review including systematic reviews and meta-analyzes of clinical trials on interventions of prehabilitation programs. Finally, this article presents the scope of the PAMP project in all its phases and its progress towards the implementation of a clinical trial of prehabilitation in Colombia through the PAMP phase II study was described. Conclusions: It is necessary to implement innovative multidisciplinary strategies that promote better results in the postoperative period of patients undergoing noncardiac surgery. [Cortés OL, Becerra-Cristancho $C P$, Hine-Sanabria AL, Vásquez-Hernández SM. Improvements in the knowledge of prehabilitation and presentation of the project: promotion of early ambulation in non-cardiac surgery patients (PAMP). MedUNAB 2016; 19(2): 142-150].

\section{Introducción}

A nivel mundial, cada año se realizan 200 millones de cirugías mayores no cardíacas, siendo la población adulta el grupo que más requiere estas intervenciones (1). Entre 2004 y 2012 hubo un incremento del $38.2 \%$ en el número de estas cirugías (226 a 312 millones/año), especialmente en países en desarrollo. En el 2012 la tasa media estimada de cirugía en el mundo fue de 4,469 operaciones por cada 100,000 personas, lo cual incrementó la inversión de US \$ 400 per cápita en el gasto total en salud. En Estados Unidos, durante
Keywords: General Surgery; Preoperative period; Postoperative period; Aged; Exercise.

\section{Resumo}

Introdução: Introdução: A pré-reabilitação é um programa multidisciplinar que combina um conjunto de intervenções (caminhada, aeróbica, treinamento muscular inspiratório, nutrição e psicologia) destinado a melhorar a capacidade funcional dos pacientes antes de fazer a cirurgia. $\mathrm{O}$ objetivo da pré-reabilitação é para reduzir as complicações que podem ocorrer durante o período pré-operatório (pré, trans e pós-operatório) em pessoas adultas e idosas. As complicações pré-reabilitação em todo o mundo é de 200 milhões e cerca de 1 milhão de doentes morrem durante os primeiros 30 dias depois de ter feito a cirurgia. A estimativa de risco antes da cirurgia identifica aos pacientes que necessitam de um acompanhamento mais intensivo e maior atenção depois da cirurgia. Embora a avaliação de risco antes da cirurgia pode melhorar a predição de mortalidade pós-operatória em cirurgia não cardíaca, pouco se sabe sobre a eficácia da pré-reabilitação como complemento na avaliação do risco para prevenir complicações e a norte pósoperatórias. Objetivo: Este documento é uma revisão da literatura sobre a prática de pré-reabilitação em pacientes cirúrgicos não cardíacos. Metodologia: Isso inclui uma revisão da sua definição e uma descrição geral do tipo de intervenções ou atividades implementadas. Resultados: Explica a evidência sobre o impacto desses programas sobre a evolução do paciente no pós-operatório; uma revisão de fato sobre a pré-reabilitação, a melhor prova da préreabilitação relatada em revisões sistemáticas e metaanálises. Conclusões: o avanço associado com a pesquisa nacional relacionada com a avaliação do estilo de vida sedentário e os fatores associados à inatividade, antes da cirurgia de pacientes não cardíacos e do estudo em progresso PAMP-Phase II (Promoção da deambulação précirúrgica) um projeto piloto de pré-reabilitação liderado pela enfermagem em pacientes agendados para cirurgia não cardíaca na Colômbia. [Cortés OL, Becerra-Cristancho CP, Hine-Sanabria AL, Vásquez-Hernández SM. Avanços no Conhecimento da Pré-reabilitação e Apresentação do Projeto Promoção da deambulação precoce em pacientes com cirurgia não cardíaca (PAMP). MedUNAB 2016; 19 (2): 142-150]

Palavras chave: Cirurgia Geral; Período Pré-operatório; Período Pós-operatório; Idoso; Exercício.

el año 2010 se realizaron 10 millones de cirugías no cardíacas, reportándose los desórdenes músculoesqueléticos (84\%), neoplasia $(61.4 \%)$, lesiones por accidente $(43.2 \%)$, y enfermedades digestivas $(36.2 \%)$ como las más frecuentes (1-3).

A pesar de los múltiples beneficios de la cirugía no cardíaca a corto y largo plazo, y de la indicación para aliviar o 'curar' numerosas enfermedades, esta representa aún riesgos de complicaciones para los individuos expuestos (4). Estos riesgos se pueden presentar en todo el periodo perioperatorio 
(pre, trans y postoperatorio) y pueden estar relacionados con el estado de salud previo, sus comorbilidades, y la capacidad funcional y física (sedentarismo) previo al procedimiento quirúrgico de cada individuo (4). Los adultos mayores, especialmente aquellos con comorbilidades como cáncer y una reserva limitada de proteínas se consideran en riesgo alto para resultados negativos en el periodo postquirúrgico $(1,4,5)$.

Las complicaciones isquémicas en el perioperatorio como muerte vascular, infarto de miocardio no fatal (IAM), paro cardíaco no fatal y accidente cerebro-vascular no fatal (ACV) son consideradas las complicaciones de mayor prevalencia en pacientes sometidos a cirugía no cardíaca (1-5). Se estima que alrededor de 3-5 millones de pacientes presentarán alguna de estas complicaciones, y anualmente, más de un millón de adultos operados muere a los 30 días en el periodo postoperatorio $(1,5)$.

Alrededor del 50\% de los adultos mayores experimentan pérdida de su funcionalidad en el postoperatorio y algunos pacientes hospitalizados gastan alrededor del 80\% del tiempo en reposo en cama. En cuanto a los pacientes que van a cirugía abdominal, se estima que un $30 \%$ presentan complicaciones en el postoperatorio, y aún en ausencia de complicaciones, la cirugía mayor se asocia con una reducción del $40 \%$ de la capacidad funcional $(5,6)$. Prolongados periodos de inactividad física asociados a la disminución de capacidad física (motora) y funcional llevan a la pérdida de la musculatura, desacondicionamiento, y complicaciones pulmonares (6). De este modo la calidad de vida de los pacientes se ve deteriorada y es posible que la inmovilidad conlleve a otras complicaciones intrahospitalarias como las caídas, las úlceras por presión y las infecciones nosocomiales $(4,6)$. Estas complicaciones prolongan el tiempo de estancia hospitalaria ocasionando mayor estrés y deterioro de la calidad del sueño, fatiga, una mayor reducción de la capacidad funcional, y mayor pérdida de la resistencia muscular $(4,6)$.

Aunque la mayoría de las intervenciones en términos de rehabilitación han sido dirigidas hacia el periodo postoperatorio, intervenciones en el preoperatorio pueden ser más efectivas (6). El conocimiento del riesgo de los pacientes que van a cirugía no cardíaca, su estado funcional y capacidad física y la exposición a posibles complicaciones en el perioperatorio nos indica la necesidad de establecer intervenciones preventivas en el periodo prequirúrgico $(4,6)$. Basados en esta evaluación es posible implementar intervenciones que mantengan y/o mejoren la capacidad funcional y física de los pacientes antes de la cirugía preparándolos para el postoperatorio, el egreso hospitalario, y la adaptación a las actividades de la vida diaria. Este proceso que involucra la evaluación del estatus funcional, una evaluación de la capacidad aeróbica, y evaluación del riesgo quirúrgico con el fin de promover el mantenimiento o la mejoría de la capacidad funcional desde el periodo preoperatorio se denomina prehabilitación $(4,6)$.

\section{¿Cuál es el origen de la prehabilitación?}

Las primeras investigaciones en prehabilitación se centraron en pacientes sometidos a cirugías electivas de tipo cardíaca, ortopédica y para tratamiento del cáncer y posteriormente fueron ampliándose a otras patologías (7, 8). Experimentos con animales (ratas) comparando el impacto de someter a un grupo a entrenamientos como correr continuamente comparado con un grupo sedentario en la resistencia/tolerancia al traumatismo demostraron una mayor tolerancia al trauma y menor mortalidad en el grupo intervenido $(8,9)$. El concepto de la prehabilitación no es un concepto nuevo en la prevención en salud, en 1946 se publicó uno de los primeros reportes en el British Medical Journal en población sana. Este reporte se centró en la necesidad de obtener hombres preparados para ir al campo de batalla, similar al entrenamiento militar para soldados y oficiales en guerra (8). Esta preparación militar incluía ejercicios de fuerza y resistencia, nutrición y educación en general. Es así como modelos similares en salud fueron implementados para prevenir las complicaciones originadas con el estrés fisiológico y sicológico que causaba la exposición a procedimientos intensos como la cirugía y así probar los beneficios de algunas de estas intervenciones $(8,9)$.

Actualmente, los procesos de mejoramiento de la capacidad funcional de los individuos, de tal forma que les permita el manejo (soporte) de un estresor como es la cirugía, se conocen como prehabilitación $(4,6,9)$. Aunque varios componentes del programa de prehabilitación son comunes a todos los tipos de cirugía, algunas intervenciones necesitan ser adaptadas de forma individual para lograr el alcance de las funciones corporales en ciertos pacientes $(10,11,12)$. Los profesionales de la salud reconocen la importancia de modificar la condición preoperatoria física, funcional, nutricional y sicológica del paciente con el fin de alcanzar una mayor resistencia y mejor capacidad funcional en el postoperatorio (6). La prehabilitación puede reducir la carga emocional limitando las complicaciones psicológicas que puedan prolongar la estancia hospitalaria y retrasar la recuperación de los pacientes. A pesar de la importancia de esta intervención en la reducción de complicaciones en pacientes expuestos a varios tipos de cirugía no cardíaca aún existe una limitada información acerca del costo beneficio de esta intervención y su impacto en el sistema de salud (4, 9-12). Aunque los beneficios de la actividad física han sido presentados en varias situaciones de discapacidad, existe limitada evidencia clínica acerca del rol de la promoción de la actividad física antes de procedimientos quirúrgicos no cardiacos en la prevención de complicaciones y mejores resultados en el postoperatorio. 


\section{¿Cuáles intervenciones pueden mejorar la capacidad física $y$ funcional de los pacientes que van a cirugía no cardíaca? y ¿Quiénes se benefician?}

El estado preoperatorio físico, nutricional, mental, así como el nivel de actividad física son factores de riesgo modificables que pueden influir en los resultados postoperatorios en pacientes a riesgo. El funcionamiento físico es un reflejo de la salud general y el impacto de varias enfermedades crónicas entre adultos y ancianos, sobre la capacidad de funcionar sin limitaciones en el transcurso de la vida diaria. El funcionamiento físico incluye la capacidad de realizar acciones básicas y actividades complejas, actividades consideradas esenciales para mantener la independencia y aquellas consideradas discrecionales que no son necesarias para una vida independiente, pero que pueden tener un impacto en la calidad de vida. Si el funcionamiento físico se encuentra deteriorado en el individuo al momento de ingresar a una cirugía, surgen las complicaciones ya mencionadas agregando a esto estancias prolongadas no solo en hospitalización, sino en cuidados intensivos que suelen ser agravadas por el reposo prolongado en cama $(4,6,12)$. Es así como surge la prehabilitación, un conjunto de intervenciones planeadas acorde con las necesidades de cada paciente, con el fin de disminuir el impacto de la cirugía en la calidad de vida y mejorar la funcionalidad del paciente en el postoperatorio inmediato, mediato y tardío $(4,6,9)$.

Aunque la caminata y ejercicio aeróbico han demostrado beneficio en el mejoramiento de la condición física prequirúrgica, programas de intervención multifactorial, incluyendo el manejo del estrés, la ansiedad y el estado nutricional, han indicado mejores resultados en pacientes con cáncer, especialmente $(7,8,12)$. A pesar de que se ha prolongado la sobrevida en la población adulta mayor entre 65-70 años, esta se encuentra expuesta a mayores problemas de salud que incrementan la posibilidad de ser sometidos a cirugía no cardíaca, y sería el grupo que recibiría el mayor beneficio de la prehabilitación $(4,8,13)$. Así mismo se beneficiarían adultos mayores con incremento de comorbilidades como el cáncer, la obesidad, diabetes, bajo estado nutricional, limitaciones cognitivas, y otras como la $\operatorname{artritis}(4,8,13)$.

Los beneficios de la prehabilitación actualmente, aunque más orientados hacia intervenciones enfocadas a la recuperación de la condición física, se recomiendan en conjunto con otras intervenciones orientadas al manejo del estado nutricional y psicológico (13). La actividad física es el común denominador en los programas reportados de prehabilitación $(4,6,13)$. Algunos autores recomiendan realizar una valoración de la capacidad funcional del paciente a través de pruebas de ejercicio cardiopulmonar como la de caminata de seis minutos (6MWT), tasa de consumo máximo de oxígeno $\left(\mathrm{VO}_{2 \text { peak }}\right)$ y el umbral anaeróbico (anaerobic threshold-AT) antes de recomendar una rutina de ejercicio en el paciente (10). De otro lado, la Organización Mundial de la Salud (OMS) aconseja la caminata de al menos 30-45 minutos/día (150 minutos/semana) durante el tiempo de espera de la cirugía, en pacientes que puedan caminar (2).

Los resultados de las pruebas de capacidad física pre quirúrgica determinarán la capacidad del paciente para así establecer la rutina de ejercicios más adecuada a su condición $(8,9)$. Ejercicios de respiración profunda y diafragmática con el incentivo respiratorio (espirómetro) y ejercicios de cómo toser, son recomendados en pacientes que van a cirugía de tipo abdominal, cardíaca y torácica (6, 9). Ejercicios aeróbicos y de resistencia, los cuales fortalecen el sistema musculo-esquelético, así como caminar, trotar, natación y ciclismo son recomendados para algunos tipos de cirugías acordes con la evaluación preliminar del paciente y sus capacidades $(7,9)$. Las cirugías de cáncer de seno, pulmón, próstata, ginecológicas, cabeza y cuello recomiendan ejercicios de equilibrio, de fortalecimiento muscular, cardiovasculares, y ejercicios de piso pélvico. En cirugías de cáncer de pulmón se recomiendan ejercicios respiratorios. Algunas cirugías oncológicas además de lo anterior incluyen actividades de ejercicio adicionales, como ejercicios de deglución (cirugías de cáncer de cabeza y cuello), y ejercicios de movilidad en brazos según el lado afectado (cirugía de cáncer de seno) $(7,8)$.

Por otra parte, el propósito de la intervención nutricional prequirúrgica es la de preparar y mantener el estado nutricional del paciente para su cirugía. La meta es proveer una adecuada nutrición para compensar el catabolismo que se dará en el postoperatorio. Las recomendaciones deben seguirse antes y después de la cirugía para obtener buenos resultados. Es indispensable revisar todas las condiciones propias del paciente que puedan afectar su metabolismo incrementando el riesgo de morbilidad y mortalidad en el período postoperatorio para así poder determinar cuál es el plan nutricional más adecuado al paciente $(8,9)$. El soporte psicológico, orientado hacia el manejo de la ansiedad y la depresión en pacientes a riesgo, puede prevenir complicaciones y estancias prolongadas (10). Las intervenciones incluidas en los planes de programas de prehabilitación reportados en la literatura forman parte de las recomendaciones basadas en la evidencia sobre actividad y ejercicio incluidas en algunas guías de actividad en salud pública para individuos sanos (U.S Centres for Disease Control and Prevention CDC; The American Collegue of Sport Medicine ACSM) (21). Las recomendaciones para adultos mayores son similares a las de los adultos (caminata 30-45 minutos al día, todos los días de la semana, al menos hasta completar 150 minutos). Así mismo la recomendación para adultos con discapacidad es muy similar a la de los adultos sanos, pero las actividades que varían de acuerdo con la capacidad y habilidad de cada individuo (21). La investigación sobre el beneficio de estas intervenciones en pacientes que van a cirugía programada 
no cardíaca debe ser promovida con el fin de evaluar su impacto en los sistemas de salud.

\section{¿Cuáles pueden ser los desenlaces evaluables con la prehabilitación?}

Para la medición del impacto de la prehabilitación se requiere tener en cuenta no solo el periodo preoperatorio en el cual esta intervención o conjunto de ellas va a ser implementada, sino también el periodo postoperatorio en el que el impacto será evaluado $(8,15)$. El plan de la prehabilitación en el periodo preoperatorio incluye el incremento de la reserva fisiológica, física, nutricional y mental, mientras que en el postoperatorio se evalúa el estado funcional, independencia, percepción y sentimientos o grado de adaptación. La evaluación del impacto de las intervenciones en las primeras tres semanas posteriores a la cirugía se dirige hacia la movilidad (tiempo de movilización temprana, metros caminados, duración de la caminata, equilibrio o agilidad), control del dolor, y manejo adecuado de los efectos secundarios del tratamiento en general (15). Entre las 6 a 8 semanas siguientes el objetivo de la evaluación debe orientarse hacia la valoración de los progresos en calidad de vida, integración a las actividades de rutina diaria y regreso al trabajo $(6,8,15)$.

El tiempo de prehabilitación en relación con el momento de la intervención quirúrgica necesita ser evaluado apropiadamente. En la mayoría de los estudios, el intervalo de tiempo ha sido propuesto de 4 a 8 semanas, con periodos cortos para los pacientes con cáncer de pulmón o cáncer abdominal y períodos largos de tiempo para las condiciones más crónicas como las cirugías de columna o las artroplastias $(8,10,15)$. Se debe tener en cuenta que, así como el comportamiento sedentario provoca un declive fisiológico rápido, los efectos de la preparación física también ocurren rápidamente, por ejemplo, las mejoras cardiovasculares pueden ser observadas a las 3 semanas de haber comenzado el entrenamiento físico, inclusive en individuos mayores $(8,10,15)$. En cuanto a la prescripción de ejercicio, hay una clara relación dosis-respuesta; entre mayor sea la actividad física, mayores serán los beneficios observados en la población observada $(8,10,15)$.

De igual forma, es importante considerar las intervenciones de tipo motivacional para que las intervenciones de nutrición y actividad física sean bien recibidas por parte de los participantes y sean incluidas dentro de sus actividades de la vida diaria al egreso hospitalario y no solamente durante el periodo preoperatorio.

Finalmente, factores como el tiempo entre la prescripción del procedimiento hasta la cirugía, las comorbilidades del paciente, el tiempo y la disposición del cuidador principal o familiar de apoyo en el programa, las barreras de acceso en la atención en salud; la distancia entre el sitio de residencia y el lugar en donde se le dan las indicaciones de actividad física a cada paciente, motivación telefónica y seguimiento son factores que deben ser tenidos en cuenta en la evaluación de la eficacia de un programa de prehabilitación.

\section{¿Cuál es el impacto de la prehabilitación reportado en revisiones sistemáticas y metaanálisis?}

Se realizó una búsqueda de las revisiones sistemáticas y metaanálisis de ensayos clínicos que han investigado acerca del impacto de la prehabilitación en el postoperatorio de pacientes de cirugía no cardíaca publicadas entre 2011 y 2016. Nuestra búsqueda incluyó las bases de datos MEDLINE (2011 a 2015), CINAHL online (2011 a 2015), HealthStar (2011-2015), LILACS (2011-2015), y EMBASE (2011-2015). Incluimos términos de búsqueda específicos ("no cardiac surgery" or "general surgery" or "abdominal surgery" or "throracic surgery" and "prehabilitation" or "preoperative care" or "exercise therapy" and "post operative complications" or "early ambulation" or "length of stay" or "early discharge"), en inglés y en español.

Se identificaron 4 estudios, publicados en idioma inglés (ninguno en español), que incluyeron ensayos clínicos aleatorizados controlados. $(14,15,18,19)$. En general todos los estudios incluyeron ensayos clínicos aleatorizados controlados con algunos estudios seudo-experimentales (total estudios incluidos: Valkenet 12; Santa Mina 21; Francis 11; Morán 9). Los tipos de cirugías incluidas fueron abdominales (gástrica alta, hígado, vejiga, colorectal, hepatectomías) y algunos artículos incluyeron cirugía cardíaca (18) y cirugía de articulaciones (15). La población total incluida en los estudios osciló entre 435 y 1,371 pacientes, con edades entre 34 y 78 años.

Las intervenciones planteadas dentro del programa de prehabilitación incluyeron ejercicios inspiratorios, estiramientos musculares $(15,18)$, caminata (movilidad) (19); ejercicios aeróbicos, resistencia muscular y combinaciones de estas modalidades (14). Algunos estudios incluyeron intervenciones nutricionales, y educación acerca de la cirugía y cuidados. La media de intervención en general ocurrió entre 1-8 semanas y la intervención se realizó en centros de ejercicio y/o en casa, previo entrenamiento para autoreporte $(14,15,18,19)$. (Tabla 1). Los resultados identificados como significativos son hallazgos reportados en al menos uno de los estudios descritos a favor del grupo intervenido comparado con el grupo control. Los ejercicios inspiratorios efectuados en el periodo prequirúrgico redujeron la morbilidad general (OR 0.59 , IC 95\%, 0.38, 0.91, p<0.001) (14). Se identificó una reducción significativa de complicaciones a favor del grupo intervenido con ejercicios inspiratorios en pacientes de cirugía cardíaca o cirugía abdominal en el estudio de Valkenet y Morán respectivamente(OR 0.27, IC 95\%, 0.13, $0.57, \mathrm{p}<0.001$; OR 0,40 , IC 95\% 0,23, 0.72, $\mathrm{p}=0.002$ ) 
Tabla 1. Resumen de desenlaces en prehabilitación: revisiones sistemáticas y metaanálisis.

\begin{tabular}{|c|c|c|c|c|}
\hline Desenlaces de los estudios & $\begin{array}{c}\text { Valkenet et } \\
\text { al. (2011) }\end{array}$ & $\begin{array}{c}\text { SantaMina et } \\
\text { al. (2014) }\end{array}$ & $\begin{array}{l}\text { Francis } \\
\text { et al. (2015) }\end{array}$ & $\begin{array}{c}\text { Moran } \\
\text { et al. (2016) }\end{array}$ \\
\hline Estancia hospitalaria & $\checkmark$ & $\checkmark$ & & $\checkmark$ \\
\hline Reducción complicaciones clínicas & $\checkmark$ & $\checkmark$ & $\checkmark$ & $\checkmark$ \\
\hline Calidad de vida & & $\checkmark$ & $\checkmark$ & \\
\hline Dolor & & $\checkmark$ & & \\
\hline Capacidad física/funcional & & $\checkmark$ & $\checkmark$ & \\
\hline Mortalidad & & & & $\checkmark$ \\
\hline Utilización servicios de salud & & $\checkmark$ & & \\
\hline
\end{tabular}

Fuente: Elaboración propia de los autores

$(14,18)$. Adicionalmente, en este estudio se identificó una reducción del tiempo de estancia hospitalaria mayor en el grupo intervenido comparado con el grupo control entre 1-8 días (media de 4 días, OR -0.54 , IC 95\%= $-0.93,-0.107, \mathrm{p}=$ $0.0014)(15)$.

Finalmente, otros resultados presentados por los estudios, aunque no son significativos, sí son relevantes en la práctica clínica y para el desarrollo futuro de proyectos de investigación. Por ejemplo, el estudio de Santa-Mina (15), reportó un incremento en la mejoría de los pacientes intervenidos comparados con el grupo control en la evaluación de la calidad de vida $(\mathrm{p} \leq 0.05)$, reducción del dolor (una mejoría del dolor) entre la 4 y 26 semana postquirúrgica, y una reducción en costos por día $\sim$ US\$ 133/día al reducir la hospitalización. Existe incertidumbre en los otros desenlaces y no está claro en estos estudios quién realiza la intervención, la frecuencia de las intervenciones en el periodo prequirúrgico y aún más, no se han evaluado las complicaciones cardiovasculares y/o muerte. Sin embargo, las revisiones sistemáticas y metaanálisis es lo más avanzado en términos de evidencia acerca del beneficio de las intervenciones enmarcadas en programas de prehabilitación.

\section{¿Qué se sabe del proyecto promoción de la ambulación precoz en pacientes de cirugía no cardíaca (PAMP)?}

Tradicionalmente se han realizado esfuerzos para mejorar el proceso de recuperación quirúrgica mediante la promoción de la movilización temprana en el período postoperatorio. El planteamiento de la prehabilitación está orientado hacia la preparación física en el preoperatorio con el fin de mejorar los resultados en el trans y el post-operatorio. Sin embargo, este tipo de intervenciones se implementa o prescribe esporádicamente no como una práctica clínica en nuestro país, y no hace parte de un plan estratégico de cuidado de pacientes que van a ser sometidos a cirugía no cardíaca.

Los avances en investigaciones sobre prehabilitación en cirugía no cardíaca han sido visibles con la implementación del estudio PAMP (Promoción de la ambulación precoz en pacientes de cirugía no cardíaca) cuyos hallazgos llevaron a la realización de las fases I y II sobre prehabilitación.

El estudio PAMP, desarrollado en el 2009, tuvo como principal objetivo la identificación del tiempo de ocurrencia de la primera caminada de pacientes mayores de 45 años en el postoperatorio de cirugía abdominal y la determinación de factores asociados a esta deambulación temprana (16). Este estudio incluyó una cohorte retrospectiva $(n=171$ historias clínicas) y una prospectiva ( $\mathrm{n}=189$ pacientes) en dos centros de cuarto nivel de atención en Colombia. Los resultados mostraron que el tiempo medio en el cual los pacientes realizaron la primera deambulación posquirúrgica intrahospitalaria fue de 45.5 horas (DS $\pm 215.26 \mathrm{~h}$ ). Los factores asociados con la movilización temprana fueron: ser sometido a cesárea $(41.4 \%, \mathrm{p}<0.001)$, haber tenido un solo procedimiento quirúrgico $(78.3 \%, \mathrm{p}=0.008)$, tener una herida tipo cero en el post quirúrgico $(91.5 \%, \mathrm{p}=0.001)$, y tener un tiempo medio en sala de recuperación postanestésica $(3.02$ horas, $\mathrm{p}<0.001)$. De otro lado, este estudio presentó que mayores tiempos quirúrgicos (1.89 horas; $\mathrm{p}=0.002$ ) y una media de edad mayor de los pacientes (51.9 años; $\mathrm{p}<0.001)$, retardaba el tiempo del inicio de la deambulación temprana. Otros factores identificados como asociados con el retardo en el tiempo de la primera caminata en el postoperatorio fueron la presencia de sistemas de drenaje en el sitio de herida quirúrgica $(40.6 \%, \mathrm{p}=0.006)$ y la media de tiempo de ayuno posoperatorio (30.13 horas, $\mathrm{p}<0.001)(16)$. 
Estos resultados impulsaron la planeación y ejecución del PAMP fase I, un estudio de corte transversal realizado posteriormente en el 2016 (PAMP Fase I cirugía nocardíaca Cardiecol). En esta fase de PAMP se planeó un estudio transversal, una encuesta a pacientes que ingresaban a cirugía no cardíaca utilizando el cuestionario internacional de actividad física (International Physical Activity Questionnaire, IPAQ). El objetivo fue determinar la prevalencia de inactividad (sedentarismo) prequirúrigica en 500 pacientes mayores de 45 años que ingresaban a cirugía programada no cardíaca, en dos centros de cuarto nivel de atención, sobre la base de sustentar un nivel de baja capacidad funcional de los pacientes al ingreso a cirugía (17). Los resultados finales de esta fase I mostraron una prevalencia de pacientes inactivos del 60\% $(n=299)$. Este mismo estudio investigó acerca de los factores determinantes de inactividad preoperatoria en este grupo de pacientes. Los factores asociados a inactividad fueron la edad mayor de 65 años, inactividad incrementada por cada 10 años de edad (OR 1.15, IC 95\%, 1.06-1.25), tener necesidad de asistencia en actividades de la vida diaria (OR 4.16 , IC 95\%, 1.41-12.2) y pacientes con diagnóstico de diabetes mellitus 2, dependientes de administración de insulina (OR 5.11, IC 95\%, 1.5-17,3). Adicionalmente, se identificó que un total de 416 pacientes $(83.2 \%)$ caminaron durante hospitalización en el postoperatorio mediato, y lo hicieron después de las 48 horas de la cirugía, aunque la mayoría de las cirugías fueran clasificadas como de bajo riesgo(17).

Estos hallazgos mostraron que los pacientes que ingresaron a cirugía no cardíaca tenían un grado importante de inactividad asociado a ciertos factores, en su mayoría modificables. Así mismo se ha observado que los pacientes se movilizan tardíamente a las 48 horas o después en el postoperatorio, a pesar de estar expuestos a cirugías de moderada-baja complejidad, lo cual puede ser considerado un riesgo para desacondicionamiento físico y complicaciones en el post operatorio.

\section{Avances en la investigación sobre prehabilitación: Proyecto promoción de la ambulación precoz en pacientes de cirugía no cardíaca (PAMP) Fase II}

Los resultados obtenidos en PAMP y PAMP Fase I fueron la base para la planeación de un proyecto de prehabilitación piloto, el estudio PAMP Fase II, actualmente en ejecución (20). El estudio PAMP fase II (año 2016-2017) es un ensayo clínico aleatorizado controlado, piloto, cuyo propósito es determinar el impacto de la prescripción de una caminata programada estandarizada prequirúrgica comparada con la intervención convencional o grupo control (no recomendación de ejercicio), en la reducción del tiempo de estancia hospitalaria y el tiempo del inicio de la ambulación en el periodo postoperatorio de pacientes programados para cirugía no cardíaca (20). Su objetivo secundario incluye la descripción de eventos cardiovasculares isquémicos como infarto agudo de miocardio (IAM), accidente cerebro vascular (ACV), mortalidad cardíaca y la incidencia de caídas durante hospitalización tanto en el grupo intervenido como en el grupo control. El diseño incluye una muestra de 500 pacientes con edad $\geq 30$ años, que ingresan a cirugía electiva/programada no-cardíaca, con capacidad cognitiva y para movilizarse diariamente, y que requieran al menos 24 horas de hospitalización postoperatoria. Previo consentimiento informado, los pacientes elegibles son aleatorizados (sistema computarizado de números aleatorios, con asignación no conocida sobres cerrados) a uno de los dos grupos; el grupo intervenido recibe una instrucción/prescripción caminata de 30 minutos al día hasta completar 150 minutos a la semana durante todo el periodo preoperatorio. Cada paciente intervenido debe diligenciar un diario de actividad la validación de la intervención se realiza con acelerómetros y pasómetros a un grupo de pacientes de ambos grupos seleccionados aleatoriamente.

Se han tamizado hasta el momento 376 pacientes y se han incluido 134, en dos centros de cuarto nivel de atención, uno en Bucaramanga y otro en Bogotá. A continuación, reportamos algunos avances de carácter descriptivo obtenidos de 61 pacientes (Tabla 2; Tabla 3). Se ha incluido una proporción equivalente de mujeres y hombres, con una edad promedio de 58.7 años (DE 10.3). Se han identificado hasta el momento ciertas variables que pueden estar relacionadas con inactividad por encima de los rangos normales como el índice de masa corporal IMC (superior a 25.0, indicador de sobrepeso), y el índice cintura cadera superior a 0.94 (IMC normal 18.5-24.9; Índice cintura cadera normal 0.71-0.84 mujeres/0.78-0.94 hombres). Así mismo se ha observado un nivel prequirúrgico de actividad moderada-baja (130-580 METS) en ambos grupos hasta el momento (METS/sem, equivalente metabólico) (Tabla 2). La mayoría de las cirugías están relacionadas con la presencia de cáncer, siendo la cirugía general y la urológica las especialidades más frecuentes (Tabla 3 ).

El estudio PAMP fase II proporcionará información relevante acerca del impacto de la realización de una preparación física de baja-moderada intensidad preoperatoria, denominada prehabilitación, en los resultados intermedios que determinan calidad en el cuidado. Estos hallazgos son importantes en el desarrollo de futuras prácticas e investigaciónes relacionadas con la prehabilitación de pacientes con problemas de salud específicos y su evaluación pronóstica (20).

\section{Conclusiones}

El periodo postoperatorio es una fase aguda que se asocia con una marcada reducción en la capacidad física y funcional, con limitaciones en la calidad de vida, especialmente en la población mayor de 65 años. Proyectos de prehabilitación física están siendo implementados en este grupo poblacional con el fin de mejorar la respuesta física y 
Tabla 2. Comparación de variables demográficas y de morbilidad entre el grupo intervención y control en el estudio PAMP Fase II (modelo de prehabilitación)

\begin{tabular}{lll}
\hline & \multicolumn{1}{c}{$\begin{array}{c}\text { Intervención } \\
(\mathrm{n}=43)\end{array}$} & \multicolumn{1}{c}{$\begin{array}{c}\text { Control } \\
(\mathrm{n}=18)\end{array}$} \\
\hline Demográficos & $59.0(10.2)$ & $58.6(10.9)$ \\
\hline Edad, media (DE) & $21.0(34.4)$ & $10.0(16.4)$ \\
\hline Sexo, Hombres, $\mathrm{n}(\%)$ & & \\
\hline Información Basal & $26.6(4.3)$ & $578.0(0-3,759)$ \\
\hline Índice de Masa Corporal, media (DE) & $817.0(0-2,097)$ & $0.92(0.76-1.03$ \\
\hline MET-min/week, mediana (RI) & $0.92(073)$ & \\
\hline Índice Cintura Cadera, mediana (RI) & & \\
\hline Antecedentes & $12.0(27.9)$ & $5.0(22.2)$ \\
\hline Cáncer & $10.0(23.3)$ & \\
\hline Hipertensión arterial & $5.0(11.6)$ & \\
\hline Enfermedad Vascular Periférica & & \\
\hline
\end{tabular}

* no hay diferencias significativas

Fuente: Elaboración propia de los autores

Tabla 3. Comparación de variables quirúrgicas entre el grupo intervenido y el grupo control en el estudio PAMP Fase II (modelo de prehabilitación)

\begin{tabular}{lll}
\hline & $\begin{array}{c}\text { Intervención } \\
(\mathbf{n}=43)\end{array}$ & $\begin{array}{c}\text { Control } \\
(\mathbf{n}=18)\end{array}$ \\
\hline Tipo de Cirugía, $\mathbf{n}(\%)$ & $9.0(20.9)$ & $8.0(27.8)$ \\
\hline General & $19.0(44.2)$ & $27.0(44.4)$ \\
\hline Urología & $26.6(4.3)$ & $2.4(3.5)$ \\
\hline Anestesia general, $\mathbf{n}(\%)$ & $2.0(1.1)$ & $12.0(66.7)$ \\
\hline Tiempo quirúrgico horas, media (DE) & $15.0(34.9)$ & \\
\hline Tipo herida, $\mathbf{n}(\%)$ & $28.0(65.1)$ & $7.0(38.9)$ \\
\hline Limpia & & $13.0(72.2)$ \\
\hline Limpia/contaminada & $11.0(25.6)$ & $3.0(16.7)$ \\
\hline Dispositivos post cirugía, $\mathbf{n}(\%)$ & $29.0(67.4)$ & \\
\hline Drenes & $3.0(7.0)$ & \\
\hline Sonda vesical & & \\
\hline Hemovac & & \\
\hline
\end{tabular}

* no hay diferencias significativas

Fuente: Elaboración propia de los autores 
metabólica que se pone a prueba durante cualquier proceso quirúrgico no cardiaco. El objetivo de la prehabilitación es el de reducir las complicaciones en el postoperatorio y facilitar el re integro de los individuos a las actividades de la vida diaria. La estrategia conocida como prehabilitación debe ser implementada por un grupo profesional de la salud multidisciplinario con un plan de cuidado que incluya estrategias de valoración, promoción de la actividad y ejercicio, seguimiento y validación de las estrategias de ejercicio y la evaluación en el postoperatorio mediato y tardío. La investigación acerca del efecto de este tipo de intervenciones, nos permitirá avanzar en la obtención de mejores resultados para nuestros pacientes, una construcción de hospitales con mayor calidad con impacto directo en nuestro sistema de salud.

\section{Financiación}

El proyecto PAMP Fase II es financiado por Colciencias a través del Programa Cardiecol en su fase II de 2015-2017.

\section{Conflicto de intereses}

Los autores manifiestan que no tienen ningún conflicto de intereses.

\section{Referencias}

1. Devereaux PJ, Mrkorada M, Sessler DI, Leslie K, AlonsoCoello P, Kurz A, Villar JC, Sigamani A, et al. Aspirin in Patients Undergoing Noncardiac Surgery. N Engl J Med 2014; 370 (16):1494-1503. Doi: 10.1056/NEJMoa 1401105

2. Weiser TG, Haynes AB, Molina G, Lipsitz SR, Esquivel MM, Uribe-Leitz T, Fu R, Azad T, Chao TE, Berry WB, Gawande AA. Size and distribution of the global volume of surgery in 2012. Bull World Health Organ 2016; 94(3):201-209F Disponible en: http://www.who.int/ bulletin/volumes/94/3/15-159293.pdf. Doi:http:// dx.doi.org/10.2471/BLT.15.159293.

3. Rose J, Chang DC, Weiser TG, Kassebaum NJ, Bickler SW. The Role of Surgery in Global Health: Analysis of United States Inpatient Procedure Frequency by Condition Using the Global Burden of Disease 2010 Framework. PLoS ONE 2014; 9(2): e89693. Doi:10.1371/journal.pone.0089693.

4. Hoogeboom TJ, Dronkers JJ, Hulzebos EH, Van-Meet eren NL. Merits of exercise therapy before and after major surgery. Curr Opin Anaesthesiol 2014; 27(2):161-166. Doi: 10.1097/ACO.0000000000000062.

5. Devereaux PJ, Chan MT, Alonso-Coello P, Walsh M, Berwanger O, Villar JC, Wang CY, Garutti RI, et al. Association between postoperative troponin levels and 30-day mortality among patients undergoing noncardiac surgery. JAMA. 2012; 307(21):2295-304. Doi: 10.1001/jama.2012.5502

6. Carli F, Charlebois P, Stein B, Feldman L, Zavorsky G, Kim DJ, Scott S, Mayo NE. Randomized clinical trial of prehabilitation in colorectal surgery. Br J Surg. 2010; 97(8):1187-97. Doi: 10.1002/bjs.7102.
7. Santa-Mina D, Scheede-Bergdahl C, Gillis C, Carli F. Optimization of surgical outcomes with prehabilitation. Appl Physiol Nutr Metab 2015; 40(9):966-9. Doi: 10.1139/apnm-2015-0084

8. Silver JK. Cancer prehabilitation and its role in improving health outcomes and reducing health care costs. Semin Oncol Nurs 2015; 31(1):13-30. Doi: 10.1016/j.soncn. 2014.11.003

9. Carli F, Scheede-Bergdahl C. Prehabilitation to enhance perioperative care. Anesthesiol Clin 2015; 33(1):17-33. Doi: 10.1016/j.anclin.2014.11.002.

10. Le Roy B, Selvy M, Slim K. The concept of prehabilitation: What the surgeon needs to know? J Visc Surg 2016; 153(2):109-12. Doi: 10.1016/j.jviscsurg.2016.01.001.

11. Le Guen M, Fischler M, Barizien N, Beaussier M. Journée monothématique de la Sfar: Préhabilitation. Anesthésie \& Réanimation 2015; 1(5):409-415.

12. Mayo NE, Feldman L, Scott S, Zavorsky G, Kim DJ, Charlebois P, Stein B, Carli F. Impact of preoperative change in physical function on postoperative recovery: argument supporting prehabilitation for colorectal surgery. Surgery 2011; 150(3):505-14. Doi: 10.1016/j.surg.2011. 07.045 .

13. Cabilan CJ, Hines S, Munday J. The Impact of Prehabilitation on Postoperative Functional Status, Healthcare Utilization, Pain, and Quality of Life: A Systematic Review. Orthop Nurs 2016; 35(4):224-37. Doi: 10.1097/NOR.0000000000000264.

14. Moran J, Guinan E, McCormick P, Larkin J, Mockler D, Hussey J, Moriarty J, Wilson F. The ability of prehabilitation to influence postoperative outcome after intra-abdominal operation: A systematic review and metaanalysis. Surgery 2016; 160(5):1189-1201. Doi: 10.1016/j.surg.2016.05.014.

15. Santa-Mina D, Clarke H, Ritvo P, Leung YW, Matthew AG, Katz J, Trachtenberg J, Alibhai SM. Effect of total-body prehabilitation on postoperative outcomes: a systematic review and meta-analysis. Physiotherapy 2014; 100(3):196-207. Doi: 10.1016/j.physio.2013.08.008.

16. Cortés OL, Vásquez SM, Ortíz LM, Castañeda AH. Factores que determinan la primera ambulación de pacientes posquirúrgicos de cirugía abdominal."En prensa". 2009.

17. Cortés OL, Moreno-Physio K, Alvarado P, Povea C, Lloyd M, Dennis R. Inactivity and Its Associated Factors in Adults Scheduled for Noncardiac Surgery: The PAMP Phase I Study. Rehabil Nurs 2016; 0:1-9. Doi:10.1002/rnj.309

18. Valkenet K, Van-De Port IG, Dronkers JJ, De Vries WR, Lindeman E, Backx FJ. The effects of preoperative exercise therapy on postoperative outcome: a systematic review. Clin Rehabil 2011; 25(2):99-111. Doi: 10.1177/0269215510380830.

19. Francis N, Luther A, Gullick G. PTU-272 Prehabilitation programms in patients undergoing abdominal surgery within enhanced recovery: a systematic review. Gut 2015; 64(1):A180-A181. Doi:10.1136/gutjnl-2015-309861.387.

20. Cortés OL, Rincón M, Povea C, Esparza M. Impacto de la prescripción de caminada antes de la cirugía de adultos que serán sometidos a cirugía electiva no-cardíaca. Proyecto Programa de Ciencia y Tecnología- Programa Cardiecol: conocimiento y acción para reducir la dimensión de la enfermedad cardiovascular en Colombia -COLCIENCIAS 2015

21. Oja P, Titze S. Physical activity recommendations for public health: development and policy context. EPMA Journal 2011; 2:253-259. 